\title{
Botanical origin, microbiological quality and physicochemical composition of the Melipona scutellaris pot-pollen ("samburá") from Bahia (Brazil) Region
}

Daiane de Jesus Oliveira, Daiane Rodrigues dos Santos, Brunelle Ramos Andrade , Andreia Santos do Nascimento , Macela Oliveira da Silva, Carize da Cruz Mercês, Cátia Ionara Santos Lucas, Samira Maria Peixoto Cavalcante da Silva , Paula Dib de Carvalho, Fabiane de Lima Silva, Letícia M. Estevinho \& Carlos Alfredo Lopes de Carvalho

To cite this article: Daiane de Jesus Oliveira, Daiane Rodrigues dos Santos, Brunelle Ramos Andrade, Andreia Santos do Nascimento, Macela Oliveira da Silva , Carize da Cruz Mercês, Cátia Ionara Santos Lucas, Samira Maria Peixoto Cavalcante da Silva, Paula Dib de Carvalho , Fabiane de Lima Silva , Letícia M. Estevinho \& Carlos Alfredo Lopes de Carvalho (2020):

Botanical origin, microbiological quality and physicochemical composition of the Melipona scutellaris pot-pollen ("samburá") from Bahia (Brazil) Region, Journal of Apicultural Research, DOI: 10.1080/00218839.2020.1797271

To link to this article: https://doi.org/10.1080/00218839.2020.1797271

2020.
Submit your article to this journal $\sqsubset$
Џll Article views: 116

\section{世 Published online: 06 Aug 2020.}

\section{View Crossmark data $₫$}

Full Terms \& Conditions of access and use can be found at https://www.tandfonline.com/action/journallnformation?journalCode=tjar20 


\title{
ORIGINAL RESEARCH ARTICLE
}

\section{Botanical origin, microbiological quality and physicochemical composition of the Melipona scutellaris pot-pollen ("samburá") from Bahia (Brazil) Region}

\author{
Daiane de Jesus Oliveira ${ }^{\mathrm{a} *}$ (ID, Daiane Rodrigues dos Santos ${ }^{\mathrm{a}}$, Brunelle Ramos Andrade ${ }^{\mathrm{a}}$, Andreia Santos do \\ Nascimento $^{\mathrm{a}}$ (D), Macela Oliveira da Silva ${ }^{\mathrm{a}}$, Carize da Cruz Mercês ${ }^{\mathrm{a}}$, Cátia lonara Santos Lucas ${ }^{\mathrm{a}}$ (D), \\ Samira Maria Peixoto Cavalcante da Silva ${ }^{a}$ (D), Paula Dib de Carvalho ${ }^{a}$, Fabiane de Lima Silva ${ }^{a}$ (ID, Letícia M. \\ Estevinho $^{\mathrm{b}}$ (D) and Carlos Alfredo Lopes de Carvalho ${ }^{\mathrm{a}}$
${ }^{a}$ Centro de Ciências Agrárias, Ambientais e Biológicas, Universidade Federal do Recôncavo da Bahia, Cruz das Almas, Bahia, Brazil; Bragançą, Bragançą, Portugal \\ ${ }^{b}$ Department of Biology and Biotechnology, Agricultural College of Bragança, CIMO-Mountain Research Center, Polytechnic Institut of
}

(Received 5 November 2018; accepted 19 February 2020)

\begin{abstract}
Melipona scutellaris Latreille, $|8| \mathrm{I}$ is of economic importance for local beekeepers, besides its relevance in the pollination of native plant species of the Brazilian Atlantic forest. Currently, data on the use of floral resources by Meliponini colonies are scarce, particularly in urban environments. We evaluated the botanical origin, the microbiological and physicochemical characteristics of pollen stored by $M$. scutellaris in colonies in an urban environment. The samples $(n=44)$ were obtained from the metropolitan region of Salvador, Bahia, Brazil, a region of intense urban and industrial activities. We identified 52 pollen types belonging to 21 botanical families. The botanical families Fabaceae, Myrtaceae, and Anacardiaceae represented most pollen types. Aerobic psychrotrophic bacteria, Bacillus spp., molds and yeasts, fecal coliforms, Escherichia coli, Staphylococcus aureus, sulfite-reducing Clostridium, and Salmonella spp. were not found in the samples. We identified and quantified fatty acids with carbon numbers varying from C4 to C20. For the physicochemical parameters, the following variations were verified: moisture $(47.3 \%$ to $55.70 \%)$, ash $(3.45 \%$ to $5.90 \%)$, protein (I0.19 to $24.02 \%), \mathrm{pH}$ (3.28 to 3.99$)$, acidity (237.20 to $557.10 \mathrm{meq} / \mathrm{kg}$ ), lipids (2.43 to $7.94 \%)$, carbohydrates ( 10.85 to $28.89 \%)$ and total energy value $(170.60$ to $216.99 \mathrm{kcal} / \mathrm{I00 \textrm {g }}$ ). Pollen stored ("samburá") by bees is a complete food and a source of nutrients with therapeutic potential. Pollen stored by M. scutellaris consists of a heterofloral pollen with physicochemical and microbiological qualities, considered safe for human consumption. Moreover, it contains linoleic and linolenic essential fatty acids making it a potential nutraceutical product.
\end{abstract}

Keywords: pollen analysis; beekeeping microbiology; fatty acid; meliponiculture

\section{Introduction}

Melipona scutellaris Latreille, $18 \mathrm{I} \mathrm{I}$, is a Meliponini species occurring in northeastern Brazil from Bahia to Rio Grande do Norte states, particularly in regions once occupied by the Atlantic Forest (Viana et al., 20I3). M. scutellaris is easily domesticated (Rodrigues et al., 2008), this is the reason that the beehives are kept in areas where intense urban and industrial growth are overtaking the Atlantic forest (Oliveira et al., 2017). Besides its relevance in the pollination of native plant species of the Brazilian Atlantic forest, M. scutellaris is of great importance to local beekeepers that, in turn, help to maintain these pollinators. Currently, data on the use of floral resources by Meliponini colonies are needed, particularly in urban environments. Studies to better understand these bees, including the use of their floral resources in various environments and the effect on products is scarce.

Meliponiculture (stingless bee cultivation) has been prominent in the Brazilian Northeast, and honey is the main marketed product (Alves et al., 20I8a; Villas-Bôas,
2018). However, pollen stored by stingless bees presents as an alternative for this beehive product, due to its bioactive compounds and complex composition (Duarte et al., 2018; Villas-Bôas, 2018). Information on quantification of pollen production of stingless bees is scarce in the literature; however, a recent study reported that the average weight of pollen pots of $M$. scutellaris was 13.96 grams, indicating the viability of cultivation of this bee for pollen production (Alves et al., 2018b).

Pot-pollen, or "samburá", or pollen stored by the stingless bee, is a result of floral pollen combination collected by worker bees with nectar and salivary enzymes from bees. It is stored and compacted in food pots (pollen-pots), different from bee bread, produced by Apis mellifera Linnaeus, 1758, which is stored in combs (Alves et al., 20l8a; Dermarderosian \& Beuther, 2005; Lima Neto et al., 2017).

According to Kroyer and Hegedus (200I), pollen stored by bees is considered a food supplement, highlighting the relevance of studies that characterize this

\footnotetext{
*Corresponding author. E-mail: daibio2I@gmail.com
} 
Table I. Origin of Melipona scutellaris pot-pollen ("samburá") samples from meliponaries in the metropolitan region of Salvador, Bahia, Brazil.

\begin{tabular}{|c|c|c|c|}
\hline Sites & Municipalities/site & $\mathrm{N}^{*}$ & Geographic Coordinates \\
\hline A & Salvador/l & 5 & S I $2^{\circ} 5 \mathrm{I}^{\prime} 32.4^{\prime \prime} ; \mathrm{W}^{\prime} 038^{\circ} 27^{\prime} 9.9^{\prime \prime}$ \\
\hline B & Salvador $/ 2$ & 7 & S $12^{\circ} 5 \mathrm{I}^{\prime} 28.3^{\prime \prime} ; \mathrm{W} 38^{\circ} 2 \mathrm{I}^{\prime} 54.3^{\prime \prime}$ \\
\hline C & Salvador/3 & 9 & S $12^{\circ} 49^{\prime} 58.7^{\prime \prime} ;$ W $38^{\circ} 22^{\prime} 27.4^{\prime \prime}$ \\
\hline D & Lauro de Freitas & 9 & S $12^{\circ} 50^{\prime} 38 . I^{\prime \prime} ; W 38^{\circ} 2 I^{\prime} \mid 2 . I^{\prime \prime}$ \\
\hline $\mathrm{E}$ & Simões Filho & 6 & S $12^{\circ} 43^{\prime} 55.5^{\prime \prime} ;$ W $38^{\circ} 23^{\prime} 51.6^{\prime \prime}$ \\
\hline $\mathrm{F}$ & Dias D'Ávila & 8 & S $12^{\circ} 32^{\prime} 28.0^{\prime \prime} ;$ W $38^{\circ} 21^{\prime} 42.3^{\prime \prime}$ \\
\hline
\end{tabular}

promising product for human food, and its consumption is stimulated by the market of natural products (Duarte et al., 2018).

Bee pollen is considered a complete food because it contains essential amino acids, carbohydrates, crude fibers, lipids, vitamins, and phenolic compounds (Feás et al., 20I2). Pollen chemical composition depends mainly on bee species, botanical and geographical origin, climatic conditions, soil type, and agricultural practices (Pascoal et al., 2014). However, there is little information on the composition, origin, and microbiological quality of pollen stored by M. scutellaris. This is the first study that determines parameters of nutritional quality, microbiological, and botanical origin.

Therefore, this study aimed to analyze the botanical origin, and microbiological and physicochemical characteristics of Melipona scutellaris pot-pollen ("samburá") from Bahia region, Brazil.

\section{Materials and methods}

\section{Sample collection}

Sampling was carried monthly between August 2014 and August 2015 in four municipalities in the metropolitan region of Salvador, the capital city of Bahia state, Brazil, a region of the Atlantic forest with intense urban and industrial activities: A (Salvador/I, $n=5$ ), B (Salvador/2, $n=7$ ), C (Salvador/3, $n=9$ ), D (Lauro de Freitas, $n=9$ ), $E$ (Simões Filho, $n=6$ ) and $F$ (Dias D'Ávila, $\mathrm{n}=8$ ) (Table I). Every sampling month, $200 \mathrm{~g}$ of bee pollen was harvested from each melliponary. Samples of stored pollen $(n=44)$, known as "samburá", were obtained from six meliponaries. We used five colonies of $M$. scutellaris by meliponary $(A-F)$, collected with disposable spatulas and nitrile gloves, placed in sterile plastic containers and properly identified, preserved in thermal bags with ice and sent to the laboratory for analysis. Upon receipt, none of the samples had signs of any visible contamination namely fermentation, spoilage or field residues.

\section{Pollen analysis}

To verify the botanical origin of stored pollen samples $(n=44)$, the samples were prepared according to the methods established by Jones and Bryant (2004). After samples were subsequently submitted to the acetolysis process according to Erdtman (1960) and up to 1000 pollen grains were consecutively counted per sample using optical microscopy as recommended by Louveaux et al. (1978). The pollen types were identified using a reference pollen collection and the specialized literature (Punt et al., 2007; Roubik \& Moreno, 1991). Pollen types were categorized according to frequency class: predominant pollen - PP ( $>45 \%$ of total grains), secondary pollen SP (16 to 45\%), important minor pollen - IMP (3 to I5\%) and minor pollen - MP $(<3 \%)$ (Louveaux et al., 1978).

\section{Microbiological determination}

To evaluate the microbiological quality of the pollen sampled from beehives $M$. scutellaris, we checked for the presence of molds and yeasts, mesophilic, and psychrotrophic aerobic bacteria, Bacillus spp., total coliforms, Escherichia coli, Staphylococcus aureus, sulfitereducing Clostridium spores, and Salmonella spp. All assays were performed in triplicate. The samples $(n=44)$ were prepared and analyzed according to the methodology described by the Association of Official Analytical Chemists (AOAC, 1989; Association of Official Analytical Chemists - 2005.03 (AOAC), 2005), (International Organization for Standardization (ISO), 2003) and Silva et al. (2010). Counts were expressed in colony forming units per gram (CFU/g) (International Organization for Standardization (ISO), 2006).

\section{Sample preparation}

Samples were prepared according to the American Public Health Association (APHA) method described in the international standards (Downes \& Ito, 200I). Thus, $25 \mathrm{~g}$ of each sample was used to prepare the first dilution $\left(10^{-1}\right)$ in $225 \mathrm{~mL}$ of $0.1 \%$, buffered Peptone water $\left(\mathrm{H}_{2} \mathrm{Op}\right)$, and the subsequent decimal dilutions $\left(\mathrm{IO}^{-2}\right.$ to $10^{-3}$ ) were prepared in $9 \mathrm{ml}$ aliquots of the same diluent.

\section{Counting microorganisms}

Growth and quantification of mesophilic and psychrotrophic aerobic bacteria were performed using standard plates count agar (PCA) (Himedia $\left.{ }^{\circledR}\right)$. Plates were incubated at $37^{\circ} \mathrm{C}$ for 48 hours for counting aerobic mesophilic bacteria and incubated at $7^{\circ} \mathrm{C}$ for 10 days for counting aerobic psychrotrophic bacteria. Growth and counts of Bacillus spp. were performed using nutrient agar medium (Himedia $\AA$ ) with sodium chloride $1.5 \%$ and incubated at $30^{\circ} \mathrm{C}$ for $48 \mathrm{~h}$. To allow the growth and counts of molds and yeasts, we used the medium DGI 8 (Biolog) and samples were incubated at $25^{\circ} \mathrm{C}$ for five days. We followed the manufacturer's instructions of CEC-Cl SimPlate (BioControl ${ }^{\circledR}$ System, Bellevue, Washington, USA) to count total coliforms and Escherichia coli, according to AOAC (2005). 
Table 2. Pollen types identified in the sample set of Melipona scutellaris pot-pollen ("samburá") from Bahia, Brazil.

\begin{tabular}{|c|c|c|c|}
\hline Family & Pollen Type & Family & Pollen Type \\
\hline$\overline{\text { Arecaceae }}$ & Cocos nucifera & Fabaceae & Fabaceae type \\
\hline Anacardiaceae & Anacardiaceae type & Fabaceae & Piptadenia \\
\hline Anacardiaceae & Spondias & Fabaceae & Senna \\
\hline Anacardiaceae & Tapirira & Fabaceae & Senna spectabilis \\
\hline Arecaceae & Arecaceae type & Gentianaceae & Coutoubea \\
\hline Asteraceae & Asteraceae type & Lauraceae & Persea americana \\
\hline Asteraceae & Bidens & Loranthaceae & Struthanthus \\
\hline Asteraceae & Vernonia & Malpighiaceae & Byrsonima \\
\hline Burseraceae & Protium & Malvaceae & Malvaceae type \\
\hline Combretaceae & Combretaceae type & Melastomataceae & Miconia \\
\hline Combretaceae & Terminalia & Melastomataceae & Tibouchina \\
\hline Erythroxylaceae & Erythroxylum & Moraceae & Moraceae type \\
\hline Euphorbiaceae & Acalypha & Moraceae & Morus \\
\hline Euphorbiaceae & Croton & Myrtaceae & Eucalyptus \\
\hline Euphorbiaceae & Euphorbiaceae type & Myrtaceae & Eugenia \\
\hline Fabaceae & Acacia & Myrtaceae & Myrcia \\
\hline Fabaceae & Caesalpinia & Myrtaceae & Psidium \\
\hline Fabaceae & Centrosema & Rubiaceae & Borreria \\
\hline Fabaceae & Chamaecrista & Rubiaceae & Rubiaceae type \\
\hline Fabaceae & Desmodium & Sapindaceae & Cupania \\
\hline Fabaceae & Leucaena & Sapindaceae & Serjania \\
\hline Fabaceae & Macroptilium & Solanaceae & Solanum \\
\hline Fabaceae & Mimosa caesalpiniifolia & Solanaceae & Solanum paniculatum \\
\hline Fabaceae & Mimosa pudica & Urticaceae & Cecropia \\
\hline Fabaceae & Mimosa quadrivalvis & Verbenaceae & Lantana \\
\hline Fabaceae & Mimosa tenuiflora & Verbenaceae & Lippia \\
\hline
\end{tabular}

Sulfite-reducing Clostridium spores were detected and counted according to ISO (2003). The initial sample suspension ( $\mathrm{l} \mathrm{mL}, 5 \mathrm{~mL}$, and $10 \mathrm{~mL}$ ) was added to pre-sterile test tubes (triplicate). The tubes were submerged in a water bath at $80^{\circ} \mathrm{C}$ for 15 minutes to inactivate the sample. Subsequently, aliquots of $0.01 \mathrm{~mL}, 0.1 \mathrm{~mL}$, and $\mathrm{I} \mathrm{mL}$ of each sample were inoculated into Petri dishes, which were then topped with iron sulfite agar medium. The plates were packed in anaerobic jars and incubated at $37^{\circ} \mathrm{C} \pm \mathrm{I}^{\circ} \mathrm{C}$ for 24 to 48 hours.

Search for Staphylococcus ssp.

Aliquots $(0.1 \mathrm{~mL})$ of each dilution were removed and inoculated on the surface of Petri dishes containing Baird-Parker medium (Himedia $($ ) ) and the plates were incubated at $37^{\circ} \mathrm{C}$ for 24 hours (Silva et al., 20l0).

Search for Salmonella ssp.

The search for Salmonella spp. was carried out using immunodiffusion 1-2- test ${ }^{\circledR}$ following the manufacturer's recommendations (AOAC, 1989).

\section{Physicochemical characterization}

For the physicochemical characterization of $M$. scutellaris pollen samples $(n=44)$, the following parameters were evaluated: moisture content (AOAC, 1995), ash content, total lipids according to the Instituto Adolfo Lutz (IAL, 2008), protein content by the Kjeldahl method (Almeida-Muradian et al., 20I2; AOAC, 1995; Kjeldahl,
1883), $\mathrm{pH}$, total acidity (Association of Official Analytical Chemists (AOAC), 1990), total carbohydrates, and total energy value (Lima et al., 20II). All analyses were performed in triplicates.

\section{Determination of fatty acids profile}

To determine the fatty acid profile, 44 "samburá" samples were used, and the analysis was performed from a sample composed of a sampling site (A-F). The fatty acid profile of samples was determined by flame ionization (GC-FID)/capillary column detection, according to the method described by Human and Nicolson (2006). A soxhlet automatic device (FOSS, Soxtec TM 2050, Höganäs, Sweden) was used for crude fat (CF) extraction (Association of Official Analytical Chemists (AOAC), 1995).

We used a Büchi fat determination system (Association of Official Analytical Chemists (AOAC), 2000). The extract was separated by gas chromatography using hydrogen as the transporter. Quantification of fatty acids was performed by the response factor using the mixture of fatty acids 37 (Supelco TM 37 Component FAME Mix) as standard. The levels of saturated, mono, and polyunsaturated fatty acids (SUFA, MUFA, and PUFA, respectively) and the content of each fatty acid were calculated automatically by the specific software Soxtec TM 2050 Automatic System, using a pre-established factor. Three independent replicates were performed, and results were presented as mean and standard deviation. 
Table 3. Frequency of predominant pollen (PP) and secondary pollen (SP) of the Melipona scutellaris pot-pollen ("samburá") from Bahia, Brazil.

\begin{tabular}{|c|c|c|c|c|}
\hline Site & NPT & Pollen type (PP and SP) & Frequency class & FRS (\%) \\
\hline$A(n=5)$ & 23 & $\begin{array}{l}\text { Mimosa caesalpiniifolia (Fabaceae) } \\
\text { Mimosa tenuiflora (Fabaceae) }\end{array}$ & $\begin{array}{l}\mathrm{PP} \\
\mathrm{PP}\end{array}$ & $\begin{array}{r}100 \\
50\end{array}$ \\
\hline$B(n=7)$ & 30 & $\begin{array}{l}\text { Mimosa caesalpiniifolia (Fabaceae) } \\
\text { Mimosa tenuiflora (Fabaceae) } \\
\text { Psidium (Myrtaceae) } \\
\text { Myrcia (Myrtaceae) } \\
\text { Mimosa quadrivalvis (Fabaceae) }\end{array}$ & $\begin{array}{l}\text { PP } \\
\text { SP } \\
\text { SP } \\
\text { PP } \\
\text { SP }\end{array}$ & $\begin{array}{r}100 \\
57 \\
57 \\
43 \\
14\end{array}$ \\
\hline$C(n=9)$ & 33 & $\begin{array}{l}\text { Myrcia (Myrtaceae) } \\
\text { Euphorbiaceae type (Euphorbiaceae) } \\
\text { Mimosa caesalpiniifolia (Fabaceae) } \\
\text { Tibouchina (Melastomataceae) } \\
\text { Cocos nucifera (Arecaceae) }\end{array}$ & $\begin{array}{l}\text { SP } \\
\text { SP } \\
\text { PP } \\
\text { SP } \\
\text { SP }\end{array}$ & $\begin{array}{r}100 \\
89 \\
89 \\
89 \\
33\end{array}$ \\
\hline$D(n=9)$ & 36 & $\begin{array}{l}\text { Mimosa caesalpiniifolia (Fabaceae) } \\
\text { Tibouchina (Melastomataceae) } \\
\text { Tapirira (Anacardiaceae) } \\
\text { Eugenia (Myrtaceae) } \\
\text { Psidium (Myrtaceae) } \\
\text { Solanum (Solanaceae) } \\
\text { Myrcia (Myrtaceae) } \\
\text { Cupania (Sapindaceae) }\end{array}$ & $\begin{array}{l}\text { PP } \\
\text { SP } \\
\text { PP } \\
\text { SP } \\
\text { PP } \\
\text { SP } \\
\text { SP } \\
\text { SP }\end{array}$ & $\begin{array}{r}100 \\
89 \\
67 \\
44 \\
44 \\
44 \\
33 \\
22\end{array}$ \\
\hline$E(n=6)$ & 26 & $\begin{array}{l}\text { Mimosa caesalpiniifolia (Fabaceae) } \\
\text { Myrcia (Myrtaceae) } \\
\text { Tapirira (Anacardiaceae) } \\
\text { Byrsonima (Malpighiaceae) }\end{array}$ & $\begin{array}{l}\text { PP } \\
\text { SP } \\
\text { SP } \\
\text { PP }\end{array}$ & $\begin{array}{r}100 \\
100 \\
100 \\
33\end{array}$ \\
\hline$F(n=8)$ & 30 & $\begin{array}{l}\text { Miconia (Melastomataceae) } \\
\text { Mimosa caesalpiniifolia (Fabaceae) } \\
\text { Myrcia (Myrtaceae) } \\
\text { Tibouchina (Melastomataceae) } \\
\text { Terminalia (Combretaceae) } \\
\text { Mimosa tenuiflora (Fabaceae) } \\
\text { Psidium (Myrtaceae) }\end{array}$ & $\begin{array}{l}\text { SP } \\
P P \\
\text { SP } \\
\text { SP } \\
\text { SP } \\
\text { SP } \\
\text { SP }\end{array}$ & $\begin{array}{l}88 \\
88 \\
88 \\
88 \\
75 \\
38 \\
38\end{array}$ \\
\hline
\end{tabular}

*NPT: Number of pollen types, Predominant pollen: $>45 \%$ of total pollen grain and Secondary pollen: $16-45 \%$ of total pollen grain. FRS: Relative frequency sample; Sites A, B and C (samples from Salvador I-3), D (samples from Simões Filho), $\mathrm{E}$ (samples from Lauro de Freitas) and F (samples from Dias D'Ávila), as described in Table I.

\section{Statistical analyses}

Mean, medians, and standard deviations were calculated using the physicochemical data were calculated. For the analysis of the botanical origin, we considered the predominant pollen ( $\mathrm{PP}=>45 \%$ of total grains) and secondary pollen ( $\mathrm{SP}=16$ to $45 \%$ ) types. All variables were tested for normal distribution using the Shapiro-Wilk test. Likewise, the Levene test was applied to verify the homogeneity of variances. To assess significant differences between pollen collection sites, the one-way analysis of variance (ANOVA) was used. The Tukey test was performed as a post-hoc test when the effect was significant. Moreover, we used the canonical discriminant analysis (CDA) to verify the relationship between fatty acids and pollen collection sites. CDA is a dimension-reduction technique that explores linear combinations that provide maximum discrimination to the mean vectors of the treatment groups (Friendly, 20I2). All 12 fatty acids were included in the CDA. The biplot was constructed for the first two canonical variables (Can I and Can2), along with the $95 \%$ confidence ellipses, for each site. For all analyses, we adopted a significance level of $5 \%(p<0.05)$. For the statistical analysis, the software "R" statistical and programming environment version 3.4.4 ( $\mathrm{R}$ Development Core Team, 2018) was used.

\section{Results}

\section{Pollen analyses}

All 44 "samburá" samples collected at the six sites in an Atlantic rainforest region with intense urban and industrial activities revealed that $M$. scutellaris visited a wide diversity of botanical species. In the pollen spectrum of the sample, 52 pollen types were identified distributed into 2I families showing the heterofloral characteristic of "samburá" samples (Table 2).

We identified 15 pollen types as predominant (PP = $>45 \%$ of total grains) and as secondary pollen (SP $=16$ to $45 \%$ of total grains), representing 12 botanical families, with Fabaceae, Myrtaceae, and Anacardiaceae showing the greatest richness of pollen types (Table 3 ). Mimosa caesalpiniifolia pollen type was present at high frequency in all samples studied and was therefore characterized as PP ( $>45 \%$ of total pollen grain). 
Table 4. Microbiological quality of the Melipona scutellaris pot-pollen ("samburá") from Bahia, Brazil.

\begin{tabular}{|c|c|c|c|c|c|c|}
\hline & \multicolumn{6}{|c|}{ Sites (Means $\pm S D$ ) } \\
\hline & $\begin{array}{c}\text { A } \\
(n=5) \\
\end{array}$ & $\begin{array}{c}B \\
(n=7)\end{array}$ & $\begin{array}{c}C \\
(n=9)\end{array}$ & $\begin{array}{c}D \\
(n=9)\end{array}$ & $\begin{array}{c}E \\
(n=6)\end{array}$ & $\begin{array}{c}F \\
(n=8)\end{array}$ \\
\hline M.A.* & $2.2 \times 10^{3} \pm 1.0$ & $17.0 \times 10 \pm 3.0$ & $15.8 \times 10 \pm 21,9$ & $<10$ & $<10$ & $20.9 \times 10 \pm 5.6$ \\
\hline P.A. & $<10$ & $<10$ & $<10$ & $<10$ & $<10$ & $<10$ \\
\hline Bacillus spp. & $<10$ & $<10$ & $<10$ & $<10$ & $<10$ & $<10$ \\
\hline M.Y. (CFU/g) & $<10$ & $<10$ & $<10$ & $<10$ & $<10$ & $<10$ \\
\hline F coliformes & $<1$ & $<1$ & $<1$ & $<1$ & $<\mathrm{I}$ & $<1$ \\
\hline Escherichia coli & $<1$ & $<1$ & $<1$ & $<1$ & $<1$ & $<1$ \\
\hline S. aureus & $<10$ & $<10$ & $<10$ & $<10$ & $<10$ & $<10$ \\
\hline S.R.C. & $<10$ & $<10$ & $<10$ & $<10$ & $<10$ & $<10$ \\
\hline Salmonella spp. & A & A & A & A & A & A \\
\hline
\end{tabular}

*A: absent; CFU/g: colony forming unit/gram; Site A, B and C (pollen samples from Salvador I-3), D (pollen samples from Simões Filho), E (pollen samples from Lauro de Freitas) and $F$ (pollen samples from Dias D'Ávila); M.A: Mesophilic aerobes (CFU/g); P.A.: Psychotropic aerobes (CFU/g); M.Y.: molds and yeasts (CFU/g); F coliforms: fecal coliforms; S.R.C.: sulfite-reducing clostridial spores in 0.0I g. SD: Standard deviation.

\section{Microbiological quality}

We did not detect the presence of Salmonella spp., spores of reducing sulfite clostridium, total coliforms, Escherichia coli, Staphylococcus aureus, aerobic psychrotrophic bacteria, Bacillus sp., molds, and yeasts (Table 4). However, we found mesophilic aerobic bacteria in $32 \%$ of the samples, with the highest average value $\left(2.2 \times 10^{3} \mathrm{CFU} / \mathrm{g}\right)$ observed for the meliponary $\mathrm{A}$ (Salvador/I, environment with anthropic influence).

\section{Physicochemical characteristics}

The physicochemical parameters of the pollen stored by M. scutellaris are presented in Table 5. Data shows that the parameters did not differ significantly among all samples collected in six sites in a region of the Atlantic rainforest, but with intense urban and industrial activities $(p>0.05)$.

\section{Fatty acid profile}

We identified 12 fatty acids, nine saturated, and three unsaturated. The most abundant among the saturated fatty acids (SFA) was capric acid (CI0: 0), ranging from $\mathrm{I} .89$ to $5.66 \mathrm{~g} / \mathrm{lO0 \textrm {g }}$ of pollen. Oleic acid (CI8: I) was the most common monounsaturated fatty acid (MUFA). The most abundant polyunsaturated fatty acids (PUFAs) were the linoleic/omega-6 (CI8: $2 \Delta 6 ; 0.50$ to $1.63 \mathrm{~g} /$ $\mathrm{l} 00 \mathrm{~g})$ and linolenic acid/omega-3 (Cl8: $3 \Delta 3 ; 0.30$ to $0.86 \mathrm{~g} / \mathrm{l} 00 \mathrm{~g})$. There were significant differences $(p<0.05)$ in pollen fatty acid contents in terms of collection sites within the urban-industrial area addressed in this study (Figure I).

Thus, we considered the significant fatty acid compositions indicated by ANOVA. The CDA and the Wilks Lambda multivariate statistical test revealed a significant difference regarding fatty acid composition between sites of sample collection $(p<0.05)$. Figure $2 A$ shows the biplot of the two canonical discriminant functions revealing a clear separation between fatty acid pollen composition from different sites. The first canonical discriminant function (Canl) explained $35.3 \%$ of the total variance and the second canonical discriminant function (Can2) explained $30.3 \%$. The first two canonical discriminant functions explained $65.9 \%$ of the total data variation, which may be considered a high percentage.

The pollen of $D$ (samples from Simões Filho) and $F$ sites (samples from Dias D'Ávila) were different from the other sites (Can I), presenting higher values for linoleic acid (CI8: $2 \Delta 6)$, linolenic acid (CI8: $3 \Delta 3$ ), palmitic acid (Cl6: 0$)$, stearic acid ( $\mathrm{Cl} 8: 0)$ and capric acid (CI0: 0). These fatty acids contributed more to Can I formation and were more important to discriminate pollen samples between the collection sites (Figure 2B). Sites $B$ and $C$ (pollen samples from different sites in Salvador) did not contribute to CDA since they are isolated from fatty acid composition vectors in the biplot, presenting negative values for C4: $0, \mathrm{C6}: 0, \mathrm{C} 8: 0, \mathrm{Cl} 4$ : 0 and C20: 0 . Using Can2, pollen of site $E$ (samples from Lauro de Freitas) differs from sites B and C (pollen samples from different sites in Salvador) in terms of oleic acid (CI8: I). The ANOVA analysis confirmed the difference observed.

\section{Discussion \\ Pollen analyses}

The Fabaceae species, specifically Mimosa genus, have morphological features such as dense inflorescences with small and gently scented flowers that attract many species of stingless bees to collect trophic resources, such as pollen and nectar, highlighting the importance of this botanic family (Carneiro-Neto et al., 2017; MaiaSilva et al., 2012). In bee pollen samples evaluated by Almeida-Muradian et al. (2005) and Estevinho et al. (20I2) pollen types of Fabaceae were also identified. In addition, the study by $\mathrm{Di}$ Marco et al. (2017) confirms the importance of species of this botanical family also as an important source of nectar for bees.

Mimosa caesalpiniifolia Benth. is a native botanical species of the Atlantic forest and is widely used as hedgerows (Santos et al., 20I I; Souchie et al., 2005).

Few studies evaluate pollen stored by $M$. scutellaris. Alves et al. (2018a) sampled bee-derived products in an 
Table 5. Physicochemical parameters of the Melipona scutellaris pot-pollen ("samburá") from Bahia, Brazil.

\begin{tabular}{|c|c|c|c|c|c|c|}
\hline & Min. & Max. & Median & $\mathrm{Cl}$ & Mean \pm SD & SEM \\
\hline $\begin{array}{l}\text { Site A } \\
\text { Moisture (\%) } \\
\text { Ash (\%) } \\
\text { Protein (\%) } \\
\text { pH } \\
\text { T.A. (meg/kg) } \\
\text { T.L. (\%) } \\
\text { T.C. (\%) } \\
\text { TEV (kcal/l00g) }\end{array}$ & $\begin{array}{r}49.38 \\
4.55 \\
13.13 \\
3.69 \\
237.20 \\
3.02 \\
12.48 \\
173.62\end{array}$ & $\begin{array}{r}53.65 \\
5.90 \\
20.81 \\
3.91 \\
474.20 \\
5.73 \\
28.19 \\
200.39\end{array}$ & $\begin{array}{r}53.29 \\
4.90 \\
13.95 \\
3.81 \\
448.20 \\
4.63 \\
21.88 \\
189.25\end{array}$ & $\begin{array}{c}{[50.33 ; 54.80]} \\
{[4.34 ; 5.66]} \\
{[11.46 ; 20.89]} \\
{[3.68 ; 3.90]} \\
{[276.90 ; 519.40]} \\
{[3.16 ; 5.72]} \\
{[11.35 ; 28.39]} \\
{[176.30 ; 200.00]}\end{array}$ & $\begin{array}{r}52.56 \pm 1.80 \\
5.00 \pm 0.54 \\
16.18 \pm 3.80 \\
3.79 \pm 0.09 \\
398.18 \pm 97.65 \\
4.44 \pm 1.03 \\
19.87 \pm 6.86 \\
188.17 \pm 9.56\end{array}$ & $\begin{array}{r}0.80 \\
0.24 \\
1.70 \\
0.04 \\
43.67 \\
0.46 \\
3.07 \\
4.27\end{array}$ \\
\hline $\begin{array}{l}\text { Site B } \\
\text { Moisture (\%) } \\
\text { Ash (\%) } \\
\text { Protein (\%) } \\
\text { pH } \\
\text { T.A. (meq/kg) } \\
\text { T.L. (\%) } \\
\text { T.C. (\%) } \\
\text { TEV (kcal/l00g) }\end{array}$ & $\begin{array}{r}52.50 \\
3.45 \\
15.23 \\
3.58 \\
370.80 \\
3.01 \\
12.48 \\
170.60\end{array}$ & $\begin{array}{r}55.41 \\
5.25 \\
20.15 \\
3.86 \\
525.40 \\
7.05 \\
21.61 \\
198.19\end{array}$ & \begin{tabular}{r|}
53.98 \\
4.50 \\
18.03 \\
3.64 \\
487.80 \\
5.23 \\
13.74 \\
191.88
\end{tabular} & $\begin{array}{c}{[53.09 ; 54.94]} \\
{[3.99 ; 5.01]} \\
{[16.47 ; 19.53]} \\
{[3.59 ; 3.77]} \\
{[422.30 ; 524.70]} \\
{[3.61 ; 6.55]} \\
{[11.76 ; 17.92]} \\
{[178.50 ; 196.50]}\end{array}$ & $\begin{array}{r}54.01 \pm 1.00 \\
4.50 \pm 0.55 \\
18.00 \pm 1.66 \\
3.68 \pm 0.10 \\
473.49 \pm 55.39 \\
5.08 \pm 1.59 \\
14.84 \pm 3.33 \\
187.49 \pm 9.69\end{array}$ & $\begin{array}{r}0.38 \\
0.21 \\
0.63 \\
0.04 \\
20.93 \\
0.60 \\
1.26 \\
3.66\end{array}$ \\
\hline $\begin{array}{l}\text { Site C } \\
\text { Moisture (\%) } \\
\text { Ash (\%) } \\
\text { Protein (\%) } \\
\text { pH } \\
\text { T.A. (meq/kg) } \\
\text { T.L. (\%) } \\
\text { T.C. (\%) } \\
\text { TEV (kcal/l00g) }\end{array}$ & \begin{tabular}{r|}
50.57 \\
4.50 \\
13.40 \\
3.44 \\
433.00 \\
4.50 \\
10.85 \\
183.36
\end{tabular} & \begin{tabular}{r|}
54.57 \\
5.40 \\
24.02 \\
3.90 \\
512.40 \\
6.05 \\
24.00 \\
202.89
\end{tabular} & $\begin{array}{r}52.86 \\
4.95 \\
17.01 \\
3.73 \\
460.80 \\
5.43 \\
16.44 \\
191.53\end{array}$ & $\begin{array}{c}{[52.10 ; 54.00]} \\
{[4.73 ; 5.15]} \\
{[15.13 ; 20.97]} \\
{[3.60 ; 3.83]} \\
{[448.50 ; 494.30]} \\
{[5.02 ; 5.74]} \\
{[14.20 ; 20.09]} \\
{[186.90 ; 196.00]}\end{array}$ & $\begin{array}{r}53.05 \pm 1.24 \\
4.94 \pm 0.27 \\
18.05 \pm 3.80 \\
3.72 \pm 0.15 \\
471.43 \pm 29.81 \\
5.38 \pm 0.47 \\
\mid 7.15 \pm 3.83 \\
|9| .49 \pm 5.9 \mid\end{array}$ & $\begin{array}{l}0.41 \\
0.09 \\
1.27 \\
0.05 \\
9.94 \\
0.16 \\
1.28 \\
1.97\end{array}$ \\
\hline $\begin{array}{l}\text { Site D } \\
\text { Moisture (\%) } \\
\text { Ash (\%) } \\
\text { Protein (\%) } \\
\text { pH } \\
\text { T.A. (meq/kg) } \\
\text { T.L. (\%) } \\
\text { T.C. (\%) } \\
\text { TEV (kcal/l00g) }\end{array}$ & $\begin{array}{r}48.38 \\
3.60 \\
11.66 \\
3.64 \\
342.00 \\
2.43 \\
15.33 \\
185.50\end{array}$ & $\begin{array}{r}54.33 \\
5.80 \\
21.44 \\
3.99 \\
51.40 \\
7.47 \\
28.19 \\
210.65\end{array}$ & $\begin{array}{r}52.65 \\
5.20 \\
18.08 \\
3.83 \\
450.60 \\
4.73 \\
17.01 \\
187.02\end{array}$ & $\begin{array}{c}{[50.86 ; 53.91]} \\
{[4.40 ; 5.56]} \\
{[14.94 ; 19.60]} \\
{[3.72 ; 3.91]} \\
{[408.00 ; 482.10]} \\
{[3.60 ; 5.80]} \\
{[15.76 ; 22.31]} \\
{[184.70 ; 198.60]}\end{array}$ & $\begin{array}{c}52.24 \pm 1.79 \\
4.98 \pm 0.76 \\
17.27 \pm 3.03 \\
3.81 \pm 0.13 \\
445.08 \pm 48.23 \\
4.70 \pm 1.43 \\
19.04 \pm 4.26 \\
191.67 \pm 9.05\end{array}$ & $\begin{array}{r}0.60 \\
0.25 \\
1.01 \\
0.04 \\
16.08 \\
0.48 \\
1.42 \\
3.02\end{array}$ \\
\hline $\begin{array}{l}\text { Site E } \\
\text { Moisture (\%) } \\
\text { Ash (\%) } \\
\text { Protein (\%) } \\
\text { pH } \\
\text { T.A. (meq/kg) } \\
\text { T.L. (\%) } \\
\text { T.C. (\%) } \\
\text { TEV (kcal/l00g) } \\
\text { Moisture (\%) } \\
\text { Ash (\%) } \\
\text { Protein (\%) } \\
\text { pH } \\
\text { T.A. (meq/kg) } \\
\text { T.L. (\%) } \\
\text { T.C. (\%) } \\
\text { TEV (kcal/l00g) }\end{array}$ & $\begin{array}{r}47.03 \\
3.60 \\
10.19 \\
3.28 \\
342.70 \\
4.80 \\
12.03 \\
191.39 \\
51.59 \\
3.55 \\
11.76 \\
3.52 \\
351.60 \\
4.77 \\
11.63 \\
181.35\end{array}$ & \begin{tabular}{r|}
54.81 \\
5.20 \\
20.97 \\
3.83 \\
513.80 \\
7.50 \\
28.89 \\
216.99 \\
55.70 \\
5.70 \\
22.95 \\
3.93 \\
557.10 \\
7.94 \\
25.80 \\
205.84
\end{tabular} & \begin{tabular}{r|}
51.98 \\
4.32 \\
15.31 \\
3.67 \\
435.65 \\
5.73 \\
20.55 \\
203.28 \\
53.35 \\
4.50 \\
17.46 \\
3.74 \\
491.50 \\
5.50 \\
16.88 \\
191.00
\end{tabular} & $\begin{array}{c}{[48.13 ; 54.58]} \\
{[3.72 ; 5.06]} \\
{[11.36 ; 20.42]} \\
{[3.39 ; 3.83]} \\
{[369.40 ; 489.10]} \\
{[5.03 ; 6.90]} \\
{[13.61 ; 28.08]} \\
{[192.60 ; 214.30]} \\
{[52.56 ; 55.11]} \\
{[3.91 ; 5.00]} \\
{[13.46 ; 20.03]} \\
{[3.60 ; 3.81]} \\
{[423.00 ; 527.00]} \\
{[5.02 ; 6.65]} \\
{[13.33 ; 21.54]} \\
{[183.60 ; 198.80]}\end{array}$ & $\begin{array}{c}51.35 \pm 3.07 \\
4.39 \pm 0.64 \\
15.89 \pm 4.32 \\
3.61 \pm 0.21 \\
429.25 \pm 57.00 \\
5.96 \pm 0.89 \\
20.85 \pm 6.90 \\
203.43 \pm 10.36 \\
53.83 \pm 1.53 \\
4.46 \pm 0.65 \\
16.75 \pm 3.93 \\
3.70 \pm 0.13 \\
475.03 \pm 62.19 \\
5.84 \pm 0.98 \\
17.43 \pm 4.91 \\
191.19 \pm 9.11\end{array}$ & $\begin{array}{r}1.25 \\
0.26 \\
1.76 \\
0.08 \\
23.27 \\
0.36 \\
2.81 \\
4.23 \\
0.54 \\
0.23 \\
1.39 \\
0.04 \\
21.99 \\
0.35 \\
1.74 \\
3.22\end{array}$ \\
\hline $\begin{array}{l}\text { Global } \\
\text { Moisture (\%) } \\
\text { Ash (\%) } \\
\text { Protein (\%) } \\
\text { pH } \\
\text { T.A. (meq/kg) } \\
\text { T.L. (\%) } \\
\text { T.C. (\%) } \\
\text { TEV (kcal/l00g) }\end{array}$ & $\begin{array}{r}47.03 \\
3.45 \\
10.19 \\
3.28 \\
237.20 \\
2.43 \\
10.85 \\
170.60\end{array}$ & $\begin{array}{r}55.70 \\
5.90 \\
24.02 \\
3.99 \\
557.10 \\
7.94 \\
28.89 \\
216.99\end{array}$ & \begin{tabular}{r|}
53.23 \\
4.70 \\
17.69 \\
3.73 \\
456.50 \\
5.31 \\
16.82 \\
191.46
\end{tabular} & $\begin{array}{c}{[52.31 ; 53.47]} \\
{[4.54 ; 4.91]} \\
{[16.11 ; 18.17]} \\
{[3.68 ; 3.77]} \\
{[434.70 ; 471.20]} \\
{[4.89 ; 5.61]} \\
{[16.51 ; 19.56]} \\
{[189.20 ; 195.00]}\end{array}$ & $\begin{array}{c}52.89 \pm 1.90 \\
4.72 \pm 0.61 \\
17.14 \pm 3.38 \\
3.72 \pm 0.15 \\
452.95 \pm 59.93 \\
5.25 \pm 1.18 \\
18.03 \pm 5.01 \\
192.08 \pm 9.83\end{array}$ & $\begin{array}{l}0.29 \\
0.09 \\
0.51 \\
0.02 \\
9.03 \\
0.18 \\
0.76 \\
1.45\end{array}$ \\
\hline
\end{tabular}


Table 5. Continued.

\begin{tabular}{|c|c|c|c|c|c|c|}
\hline Legislation & Brazil & Argent. & France & Alves et al. (20|8a) & & \\
\hline Moisture (\%) & Max.30 & Max. 8 & Max. 6 & $44.7 I \pm 9.83$ & - & - \\
\hline Ash (\%) & Max. 4 & Max. 4 & 2 à 6 & $1.84 \pm 0.12$ & - & - \\
\hline Protein (\%) & Min. 8 & 15 à 28 & 10 à 41 & $23.88 \pm 0.10$ & - & - \\
\hline $\mathrm{pH}$ & 4 à 6 & 4 à 6 & - & $3.75 \pm 0.005$ & - & - \\
\hline T.A. (meq/kg) & Max.300 & - & - & $150.57 \pm 0.40$ & - & - \\
\hline T.L. (\%) & Min. 1.8 & - & 1 à 10 & $4.25 \pm 0.10$ & - & - \\
\hline T.C. (\%) & - & - & - & $24.48 \pm 10.10$ & - & - \\
\hline TEV (kcal/l00g) & - & - & - & $231.33 \pm 37.83$ & - & - \\
\hline
\end{tabular}

*T.A.: total acidity; T.L.: total lipids; T.C.: total carbohydrates; TEV: total energy value; Max: maximum; Min: minimum; Cl: $95 \%$ confidence interval; SD: standard deviation; SEM: standard error of the means.

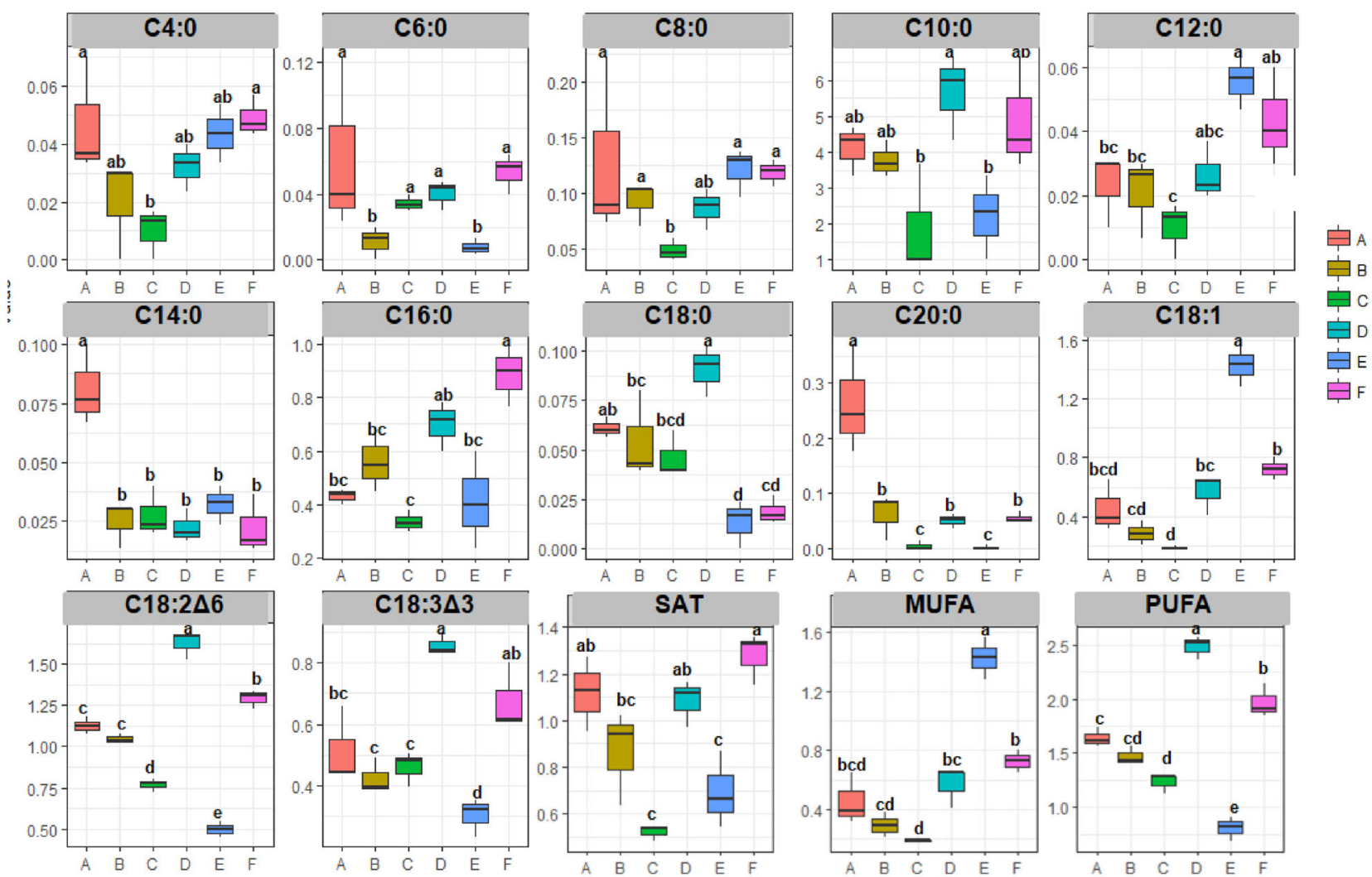

Figure I. Fatty acid profile $(\mathrm{g} / \mathrm{l} 00 \mathrm{~g})$ of the Melipona scutellaris pot-pollen (Samburá) from Bahia, Brazil. A, B, C (pollen samples from Salvador), D (pollen samples from Simões Filho), E (pollen samples from Lauro de Freitas) and $F$ (pollen samples from Dias D'Ávila). For each fatty acid, different lowercase letters $(a e)$ indicate significant differences $(p<0.05)$. Butyric acid (C4: 0$)$; caproic acid (C6: 0$)$;

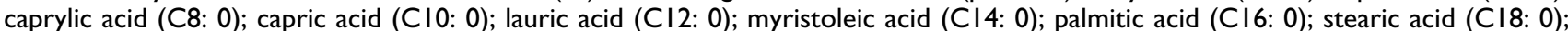
arachidic acid (C20: 0); oleic acid (CI8: I); $\alpha$-linolenic acid (CI8: $3 \Delta 3$ ); linoleic acid (CI8: $2 \Delta 6)$; SAT: total saturated fatty acid; MUFA: total of monounsaturated fatty acid; PUFA: total of polyunsaturated fatty acid.

anthropic area close to sites (Table I, Sites D and F) studied here and found seven pollen types in common with our samples Eugenia, Miconia, M. caesalpiniifolia, Myrcia, Psidium, Solanum and Tapirira. Oliveira et al. (2017) also studied an anthropic area (with transitional vegetation of a seasonal forest/Caatinga) and found three pollen types in common to those reported here, that is, M. caesalpiniifolia, Psidium and Solanum. The species Byrsonima, Eugenia, Miconia, Mimosa tenuiflora, Myrcia, Psidium, and Solanum were also found in pollen loads of $M$. scutellaris corbiculae in colonies established in a coffee plantation (Lucas et al., 2018). Previous studies on different products (geopropolis and honey) reported the presence of $M$. caesalpiniifolia pollen type, proving to be a source of the trophic resource of apicultural potential (Barros et al., 2013; Nascimento et al., 2015).

Similar pollen types were also found in studies on other Melipona species. Luz et al. (20II) studied Melipona capixaba and reported six pollen types (Cupania, Eugenia, M. caesalpiniifolia, Myrcia, Solanum and Tapirira) in common with our work. Pollen from M. caesalpiniifolia and $M$. tenuiflora were also found in samples of Melipona mandacaia (Carneiro-Neto et al., 2017). 

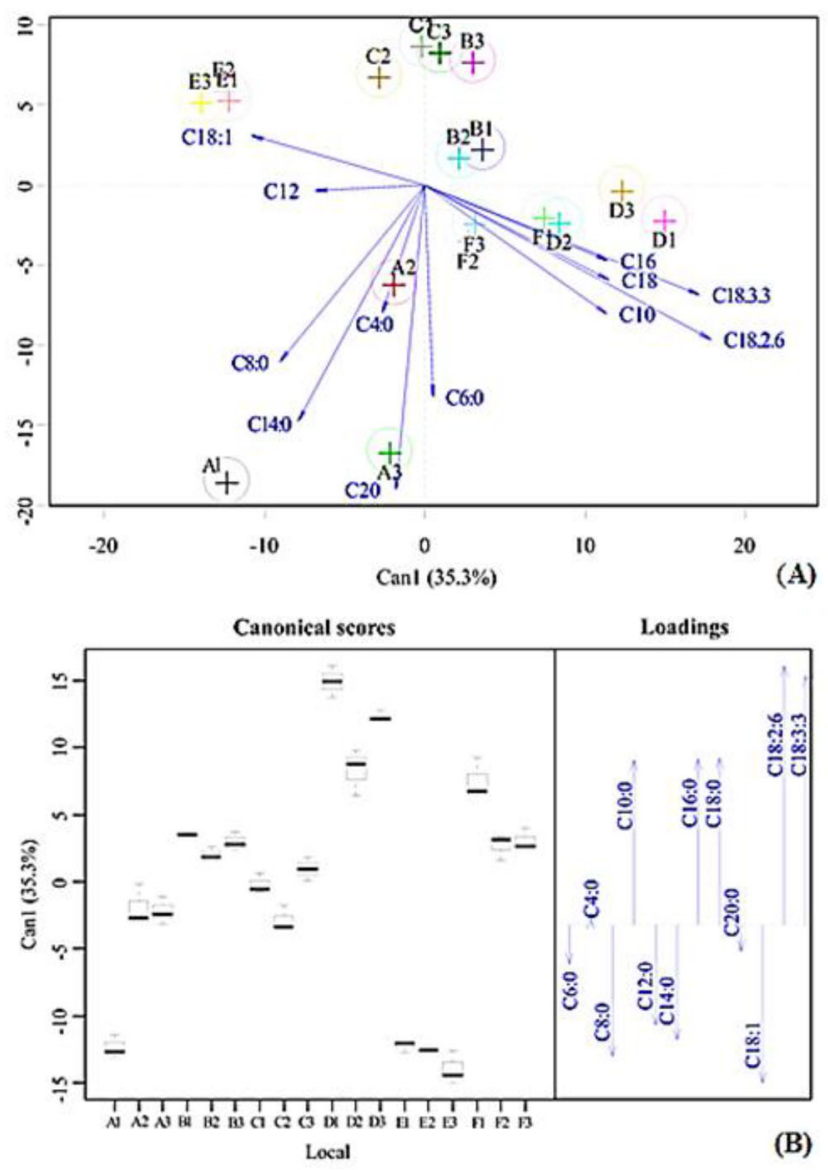

Figure 2. Canonical discriminant analysis of the relationship between the fatty acid composition and the different sites of an urbanized area where pot-pollen (samburá) by Melipona scutellaris was collected. (A) Biplot of the canonical variables (Can I, Can2). Butyric acid (C4: 0); caproic acid (C6: 0); caprylic acid (C8: 0); capric acid (Cl0: 0); lauric acid (Cl2: 0); myristoleic acid (Cl4: 0); palmitic acid (Cl6: 0); stearic acid (Cl8: 0); arachidic acid (C20: 0); oleic acid (CI8: I); $\alpha$-linolenic acid (CI8: $3 \Delta 3$ ); linoleic acid (Cl8: $2 \Delta 6)$. (B) Boxplot of the scores of the canonical discriminant variable representing the origin of the pollen. Sites $A, B$ and $C$ (pollen samples from Salvador I-3), D (pollen samples from Simões Filho), E (pollen samples from Lauro de Freitas) and $F$ (pollen samples from Dias D'Ávila).

Thus, for M. scutellaris, the data available reveal pollen from Psidium and Solanum in all studies, while $M$. caesalpiniifolia, Eugenia, Miconia, and Myrcia are found in most, but not all samples. However, of all pollen types, M. caesalpiniifolia stands out, since pollen from this botanical species was found as PP in all samples of all six sites investigated in this study as well as in samples of other two different Melipona species. The pollen types found in the "samburá" samples suggest that these botanical species are a supply of protein resource for feeding and maintaining colonies of this genus of stingless bees.

The pollen spectrum of bee pollen samples from a region composed primarily of a range of tropical forest with mangroves evaluated by Alves and Santos (2014) revealed similar preferences of these social bees (A. mellifera and M. scutellaris) in the search of protein resources, being that pollen types Cocos nucifera, Myrcia and representatives of the Mimosa genus were the most frequent. In our study, these pollen types contributed to the composition of the samburá pollen spectrum (Tables 2 and 3).

\section{Microbiological quality}

Pollen is consumed in natura and, despite its benefits to human health, its consumption requires caution since it is susceptible to environmental contaminants and growth of microorganisms. Thus, safety is a relevant aspect to be considered in this food. This result was higher than that found by Alves et al. (20l8a) when analyzing pollen from $M$. scutellaris from an Atlantic forest region and similar to those of Santa-Bárbara et al. (2015) studying pollen of M. mandacaia Smith, 1983.

Possibly, different sources of contamination available at the site and the management of the colonies favored contamination in samples of meliponary A (Salvador/l, environment with anthropic influence), since the occurrence of mesophilic bacteria is related to the conditions of food processing and storage (DeMelo et al., 2016; Feás et al., 2012). Brazilian legislation does not regulate the hygienic parameters for bee pollen (Brasil, 200I). However, the samples analyzed possibly do not pose a risk to human consumption, since the levels of mesophilic aerobic bacteria are below the recommended level indicated for food 
safety by the Argentinian Food Code (below $1.5 \times 10^{5}$ CFU/g) (De Arruda et al., 2017).

\section{Physicochemical characteristics}

Pollen stored by stingless bees is agglomerated by the addition of saliva and honey, thereby acquiring pasty consistency and it is stored in wax pots that favor the maintenance of moisture (Alves et al., 2018a). Rebelo et al. (2016) found a mean value for the moisture of $53.39 \pm 0.50 \%$ for $M$. seminigra Cockerell, 1919, similar to the result found in our study. Zuluaga et al. (20I5) in a study of the physicochemical composition of bee bread (A. mellifera) recorded an average value of $15.6 \pm 3.60 \%$ for this parameter. These results demonstrate that fermented pollen collected by stingless bees presents higher moisture when compared to bee bread. This difference is possibly related to environmental conditions of the site where the samples were collected since pollen can absorb the moisture of the environment, as it is a hygroscopic product (Marchini et al., 2006). High amounts of moisture contribute to pollen fermentation, a peculiar characteristic of "samburá" of stingless bees (Rebelo et al., 2016).

The $\mathrm{pH}$ values were similar to those observed by Alves et al. (20l8a). Lower $\mathrm{pH}$ values were observed in pollen from Melipona (Santa-Bárbara et al., 2015) and A. mellifera (De-Melo et al., 2016). The range found for total acidity (237.20 to $557.10 \mathrm{meq} / \mathrm{kg}$ ) was very different from that found in other studies with $M$. scutellaris and other Meliponini species (Almeida-Anacleto et al., 2009; Alves et al., 20l8a). The lower $\mathrm{pH}$ and high acidity values found in this work may be related to the chemical reactions that occur during the pollen fermentation process, promoted by microorganisms (Ellis \& Hayes, 2009; Kalaycıoglu et al., 2017). Moreover, the botanical origin of this product may contribute to these results (Alves et al., 20I8a).

The ash content of the samples evaluated presented an average value of $4.72 \%$, which was higher than the ash content values reported in other studies (Alves et al., 20l8a; Rebelo et al., 2016; Santa-Bárbara et al., 2015). The ash content expresses the minerals in pollen (Abadio Finco et al., 20l0; Pita-Calvo \& Vázquez, 2017) and ash values above the threshold established in Brasil (200I) may also be associated to inorganic contaminants in the environment (Kostic et al., 20I5), which may show that our samples came from an urban and industrial area. Moreover, the relatively high ash content in the samples may be attributed to the different geographical conditions where the hives were located (Abadio Finco et al., 2010; Pita-Calvo \& Vázquez, 2017).

The ash content is an important parameter, reflecting the abundance of minerals in the bee pollen sample, which indicates the need for monitoring the content of potentially toxic metals that may compromise the quality of this beehive product. Indeed, the presence of metals was verified in both honey and pollen in urban environments (Nascimento et al., 2018; Porrini et al., 2003).

The values of protein content in this work show an average of $17.14 \%$, lower than that found by Alves et al. (2018a) (23.88\%). In other studies on Melipona, SantaBárbara et al. (2015) and Rebelo et al. (2016) obtained higher values $(21.00 \%$ and $24.00 \%)$, respectively. These differences may be related to different compositions of flora and edaphic conditions.

Lipids, such as fatty acids, sterols, and triglycerides, are of great importance in the food industry as they represent energy source in the diet and, consequently, directly affect the food nutritional values. Variation in the lipid content in pollen samples studied here $(2.43$ to 7.94\%) was close to that obtained by Alves et al. (20l8a) (3.97\% to 4.46\%). However, Souza et al. (2004) studied pollen stored by stingless bees from the Amazonian region Melipona compressipes Smith, 1854, M. rufiventris Lepeletier 1836, and $M$. seminigra Cockerell, 1919, and reported a large variation in lipid content (I.9 to $9.3 \%$ ). This wide variation may be due to plant species available at the sites (Yang et al., 20/3), which is likely to be more diverse than that found in the areas where our samples were collected due to the effect of urbanization and industrialization in these areas and, hence, the narrower variation observed here.

We observed that, although the pollen evaluated was obtained from an urban area (Table I) with vegetation stratification, the results obtained for lipids and fatty acids in our study are similar to those recorded by other authors for Melipona (Alves et al., 2018a; SantaBárbara et al., 2015) and A. mellifera (Araújo et al., 2017), signaling the nutritional potential of pollen stored by $M$. scutellaris. As indicated by the bee behavior already described in literature that refers to the search of trophic resources in plant species with higher nutritional values (Filipiak et al., 2017; Newstrom et al., 1994; Silva et al., 2007).

Carbohydrates are important indicators of nutritional value and energy content, corresponding to sugars, starch, and fibers, and can comprise $2 / 3$ of the total pollen constituents (Carpes et al., 2009). The mean value for total pollen carbohydrates of $M$. scutellaris was $18.03 \%$, similar to that found in other studies on pollen of $M$. scutellaris, $M$. seminigra and $M$. interrupta Latreille, I8I I (Alves et al., 20I8a; Rebelo et al., 2016). High values of carbohydrates in pollen are attributed to the presence of honey or nectar used by bees to compact the grains (Kostić et al., 20I5).

The energy value is directly associated with the number of macronutrients accumulated in a given food (Souza et al., 2004). In this sense, the value obtained for total energy value (TEV) $(192.08 \pm 9.83 \mathrm{kcal} / \mathrm{l00 \textrm {g }})$ in the pollen analyzed here indicates that this beehive product has the potential to be used as a food supplement because it is a source of macronutrients (carbohydrates, proteins and lipids) for humans. These results also 
suggest that the flora used by $M$. scutellaris in urban areas has the essential nutritional and energetic requirements to ensure the health of these pollinators. TEV of the samples studied was lower than that found by Alves et al. (20I8a) and Rebelo et al. (20I6). For pollen in natura of $A$. mellifera, Barreto et al. (20/2) found an average total energy value of $174.7 \mathrm{kcal} / 100 \mathrm{~g}$. This variation in TEV values reported by different studies may be related to the period of sample collection, bee species, edaphoclimatic conditions, as well as the geographic and botanical origin of stored pollen, which is an important factor considering that the composition of this product is directly related to its floral origin (Feás et al., 2012; Pascoal et al., 20I4).

Possibly, the flora found at these collection sites does not change very much; however, a specific study is required to investigate that and it is beyond the scope of this study.

\section{Fatty acid profile}

Our study is the first to investigate the composition of fatty acids in pollen stored by $M$. scutellaris. For bees, fatty acids play an essential role in reproduction, development, and growth of these insects (Mărgăoan et al., 2014). Some fatty acids, such as linoleic, linolenic, myristic, and lauric have bactericidal action and antifungal properties that are important for colony hygiene (Yang et al., 20l3).

There is variability in fatty acids of pollen samples stored by bees. Other authors evaluated the dehydrated pollen of M. mandacaia and commercial pollen of A. mellifera in an agro-industrial area and identified linoleic acid (CI8:2 $\Delta 6)$ as the most abundant among the PUFAs (Dong et al., 2016; Santa-Bárbara et al., 20I5).

Considering that the pollen samples evaluated here were heterofloral, with the contribution of several plant species (with 52 pollen types identified), this variability in the number of fatty acids identified $(n=12)$ is probably related to the place of origin, urban environment and, consequently, anthropogenic influence. Due to the lower abundance of individuals of the same plant species in urban areas, bees seek their trophic resources in different plants. The botanical origin affects composition as well as chemical and sensorial characteristics of the stored pollen (Agostini \& Sazima, 2003; Aleixo et al., 20I3; Faria et al., 20I2).

The polyunsaturated fatty acids (PUFAs) of major relevance in pollen are linolenic, linoleic, and oleic acids (Kaplan et al., 2016). These acids have anti-atherogenic and anti-thrombogenic effects (Garaffo et al., 20l I) and play an important role in human metabolism, such as decreasing the levels of triacylglycerol, blood pressure, insulin resistance, and cardiovascular problems (Ghaeni et al., 20/3). PUFAs cannot be synthesized by the human body and, therefore, consumption of bee pollen can be considered a source of these acids for human diet (Kaplan et al., 2016).
A nutritional source rich in fatty acids is essential for bees because they are important for the structural integrity and functioning of the membranes of these insects (Somerville, 2005). Particularly, fatty acids are very important for bees, being metabolized mainly during the larval stages and are seen as a relevant source of energy, as well as precursors of other biosynthesis (Cantrill et al., I98I). Thus, it is suggested that the heterofloral pollen collected by $M$. scutellaris in an urban environment meets the nutritional requirements of this bee's species.

The diversity of floral species of the region is the most influential factor for the quality and chemical composition of "samburá". Sites D (samples from Simões Filho) and F (samples from Dias D'Ávila) presented the highest diversity of pollen species, including Fabaceae, Myrtaceae, and Anacardiaceae.

\section{Innovation and relevance}

This study is the first to determine the profile of fatty acids in pollen stored by the stingless bee $M$. scutellaris collected in six sites located in an urban-industrial region that was once occupied by the Atlantic forest in Brazil. Our data showed the predominance of linoleic acid (omega-6) in the samples. This fatty acid is considered important in the anti-atherogenic effect. Physicochemical, nutritional, and microbiological results presented here suggest that pollen stored by $M$. scutellaris may be consumed without risks to human health, revealing it as a promising nutraceutical product. Our data might be of interest to Brazilian food safety agencies, as they develop regulations for microbiological and physicochemical quality criteria for pollen stored by bees such as $M$. scutellaris.

The findings presented here might facilitate the activity of rearing stingless bees, making meliponiculture a potentially sustainable activity. The presence of colonies of $M$. scutellaris in urban-industrial areas may contribute to the ecosystem service in maintaining biodiversity and preserving the species as well as providing an income for beekeepers of stingless bees.

\section{Conclusions}

The bee pollen produced by $M$. scutellaris in the urbanindustrial area is heterofloral, and $M$. caesalpiniifolia is the main plant resource used by these bees. "Samburá" presented good microbiological conditions and physicochemical quality in addition to essential fatty acids, such as linoleic and linolenic acid, making it a nutraceutical product.

\section{Disclosure statement}

No potential conflict of interest was reported by the author(s). 


\section{Funding}

This study was financed in part by the "Coordenação de Aperfeiçoamento de Pessoal de Nível Superior - Brasil" (CAPES) - Finance Code $00 \mathrm{I}$ and by the "Fundação de Amparo à Pesquisa do Estado da Bahia" (FAPESB) - Finance Code PAM0004/2014. We thank "Conselho Nacional de Desenvolvimento Científico e Tecnológico" (CNPq) for the scholarship granted to CALC (No. 305885/2017-0).

\section{ORCID}

Daiane de Jesus Oliveira (iD http://orcid.org/0000-000 I7734-0693

Andreia Santos do Nascimento (D) http://orcid.org/0000-000 I5236-0460

Cátia lonara Santos Lucas (ID http://orcid.org/0000-000 I8975-4703

Samira Maria Peixoto Cavalcante da Silva (D) http://orcid.org/ 0000-000 I-8275-4575

Fabiane de Lima Silva (D) http://orcid.org/0000-0002-7262-9225

Letícia M. Estevinho (D) http://orcid.org/0000-0002-9249-1948

Carlos Alfredo Lopes de Carvalho (D) http://orcid.org/0000-00023306-3003

\section{References}

Abadio Finco, F. D. B., Moura, L. L., \& Silva, I. G. (20I0) Propriedades físicas e químicas do mel de Apis mellifera $L$. Ciência e Tecnologia de Alimentos, 30(3), 706-7/2. https://doi. org/I0.1590/SOIOI-206/2010000300022

Agostini, K., \& Sazima, M. (2003). Resources of ornamental plants for bee on campus of the State University of Campinas, São Paulo, Brazil. Bragantia, 62(3), 335-343. https://doi.org//0.1590/S0006-8705200300030000 I

Aleixo, K. P., Faria, L. B., Garófalo, C. A., Imperatriz-Fonseca, V. L., \& Silva, C. I. (20I3). Pollen collected and foraging activities of Frieseomelitta varia (Lepeletier) (Hymenoptera: Apidae) in an urban landscape. Sociobiology, 60(3), 266-276. https://doi.org// 0.13102/sociobiology.v60i3.266-276

Almeida-Anacleto, D., Marchini, L. C., Moreti, A. C. C. C., \& Souza, V. C. (2009). Food resources used by Meliponini (Hymenoptera: Apidae) in Piracicaba country, State of São Paulo, Brazil. Sociobiology, 54, 925-937. https://doi.org//0. 13102/sociobiology.v60i4.436-440

Almeida-Muradian, L. B., Arruda, V. A. S., \& Barreto, L. M. R. C. (20I2). Manual de Controle de Qualidade do pólen apícola (p. 28). Apacame.

Almeida-Muradian, L. B., Pamplona, L. C., Coimbra, S., \& Barth, O. M. (2005). Chemical composition and botanical evaluation of dried bee pollen pellets. Journal of Food Composition and Analysis, I8(I), 105-III. https://doi.org/I0. 1016/j.jfca.2003.10.008

Alves, R. F., \& Santos, F. A. R. (20/4). Plant sources for bee pollen load production in Sergipe. Palynology, 38(I), 90-100. https://doi.org//0.1080/01916122.2013.846280

Alves, R. M. O., Santos, D. R., Nascimento, A. S., Sodré, G. S., \& Carvalho, C. A. L. (2018b). Heritability of biometric parameters of Melipona scutellaris Latreille, |8I| (Hymenoptera: Apidae). Pubvet, 12(12), 1-7. https://doi.org/ 10.31533/pubvet.v12n 12a229.1-7

Alves, R. M. O., Sodré, G. S., \& Carvalho, C. A. L. (2018a). Chemical, microbiological, and palynological composition of the "Samburá" melipona scutellaris pot-pollen. In P. Vit, S.R.M. Pedro, \& D. Roubik (Eds.), Pot-pollen in stingless bee melittology. Springer, Chaper 25.

Araújo, J. S., Chambó, E. D., Costa, M. A. P. C., Silva, S. M. P. C., Carvalho, C. A. L., \& Estevinho, L. M. (2017). Chemical composition and biological activities of mono- and heterofloral bee pollen of different deographical origins. International Journal of Molecular Sciences, 18(5), 921-936. https://doi.org//0.3390/ijms I805092 I

Association of Official Analytical Chemists - 2005.03 (AOAC). (2005). Detection and confirmed quantitation of coliforms and $E$. coli in foods SimPlate coliform and E. coli color indicator first action. Association of Official Analytical Chemists.

Association of Official Analytical Chemists - 920.39 and 930.09 (AOAC). (2000). Official methods of analysis (17th ed.). AOAC International.

Association of Official Analytical Chemists - 984.13 (AOAC). (1995). Official methods of analysis (16th ed.). Association of Official Analytical Chemists.

Association of Official Analytical Chemists - 989.13 (AOAC). (1989). Motile Salmonella in All Foods Immunodiffusion (I-2 Teste). Method First Action. Association of Official Analytical Chemists.

Association of Official Analytical Chemists (AOAC). (1990). Official method of analysis of the AOAC (15th ed.). Association of Official Analytical Chemists Inc.

Barreto, L. M. R. C., Nordi, J. C., Dib, A. P. S., César, V. S., Alvareli, L. G., Nordi, N. T., \& Canella, J. B. (20/2). Physico-chemical quality of the bee pollen produced in the Paraíba Valley-SP. Revista Biociências, 18, 64-70.

Barros, M. M. R., Luz, C. F. P., \& Albuquerque, P. M. C. (2013). Pollen analysis of geopropolis of Melipona (Melikerria) fasciculata Smith, 1854 (Meliponini, Apidae, Hymenoptera) in areas of Restinga, Cerrado and flooded fields in the state of Maranhão. Grana, 52(2), 8I-92. https:// doi.org/I0.1080/00173134.2013.765909

Brasil. (200I). Ministério da Saúde. Agência Nacional de Vigilância Sanitária. Legislação. Instrução Normativa n.3, de 19 de janeiro de 2001. Aprova os Regulamentos Técnicos de Identidade e Qualidade de Apitoxina, Cera de Abelha, Geleia Real, Geleia Real Liofilizada, Pólen Apícola, Própolis e Extrato de Própolis.

Cantrill, R. C., Hepburn, H. R., \& Warner, S. J. (198I). Changes in lipid composition during sealed brood development of African worker honeybees. Comparative Biochemistry and Physiology Part B: Comparative Biochemistry, 68(2), 35I-353. https://doi.org/ I 0.1016/0305-0491(8I)901 I I-5

Carneiro-Neto, T. F. S., Rebouças, P. O., Pereira, J. E., Duarte, P. M., Santos, M. H. L. C., Silva, G. C., \& Siqueira, K. M. M. (2017). Spectrum of Pollen Stored by Melipona mandacaia (Smith, 1863) (Hymenoptera: Apidae, Meliponini) in an Urban Arid Landscape. Sociobiology, 64(3), 284-29I. V 64i3.1257. https://doi.org//0.13102/sociobiology

Carpes, S. T., Cabral, I. S. R., Rosalen, P. L., Alencar, S. M., \& Masson, M. L. (2009). Caracterização do potencial antimicrobiano dos extratos de pólen apícola da região sul do Brasil. Alimentos e Nutrição, 20, 27I-277.

De Arruda, V. A. S., Santos, A. V., Sampaio, D. F., Araujo, E. S., Peixoto, A. L. C., Estevinho, M. L. F., \& AlmeidaMuradian, L. B. (2017). Microbiological quality and physicochemical characterization of Brazilian bee pollen. Journal of Apicultural Research, 56(3), 231-238. https://doi.org//0.1080/ 00218839.2017 .1307715

De-Melo, A. A. M., Estevinho, M. L. M. F., Sattler, J. A. G., Souza, B. R., Freitas, A. S., Barth, O. M., \& Almeida-Muradian, L. B. (2016). Effect of processing conditions on characteristics of dehydrated bee-pollen and correlation between quality parameters. LWT - Food Science and Technology, 65, 808-815. https:// doi.org/I0.1016/j.lwt.2015.09.014

Dermarderosian, A., \& Beuther, J. A. (2005). Review of natural products: Facts and comparisons (4th ed., p. 1343). Wolters Kluwer Health Inc.

Di Marco, G., Manfredini, A., Leonardi, D., Canuti, L., Impei, S., Gismondi, A., \& Canini, A. (2017). Geographical, 
botanical and chemical profile of monofloral Italian honeys as food quality guarantee and territory brand. Plant Biosystems - An International Journal Dealing with All Aspects of Plant Biology, I5I(3), 450-463. https://doi.org/I0.1080/ II263504.2016.1179696

Dong, J., Yang, Y., Wang, X., \& Zhang, H. (2016). Fatty acid profiles of 20 species of monofloral bee pollen from China. Journal of Apicultural Research, 54(5), 503-5II. https://doi. org/I0.1080/00218839.2016.1173427

Downes, F. P., \& Ito, K. (200I). Compendium of methods for the microbiological examination of foods (4th ed., p. 676). American Public Health Association (APHA).

Duarte, A. W. F., Vasconcelos, M. R. S., Oda-Souza, M., Oliveira, F. F., \& López, A. M. Q. (2018). Honey and bee pollen produced by Meliponini (Apidae) in Alagoas, Brazil: Multivariate analysis of physicochemical and antioxidant profiles. Food Science and Technology, 38(3), 493-503. https://doi.org//0.1590/fst.09317

Ellis, A. M., \& Hayes, G. W. Jr. (2009). An evaluation of fresh versus fermented diets for honey bees (Apis mellifera). Journal of Apicultural Research, 48(3), 215-216. https://doi. org/I0.3896/IBRA. I.48.3.I I

Erdtman, G. (1960). The acetolysis method. A revised description. Svensk Botanisk Tidskrift, 54, 56I-564.

Estevinho, L. M., Rodrigues, S., Pereira, A. P., \& Feás, X. (2012). Portuguese bee pollen: palynological study, nutritional and microbiological evaluation. International Journal of Food Science \& Technology, 47(2), 429-435. https://doi.org/ I0.I I I I/j. I 365-262I.20II.02859.x

Faria, L. B., Aleixo, K. P., Garófalo, C. A., Imperatriz-Fonseca, V. L., \& Silva, C. I. (20I2). Foraging of Scaptotrigona aff. depilis (Hymenoptera, Apidae) in an urbanized area: Seasonality in resource availability and visited plants. Psyche: A Journal of Entomology, 2012, I-12. https://doi.org//0.1/55/2012/ 630628

Feás, X., Vázquez-Tato, M. P., Estevinho, L., Seijas, J. A., \& Iglesias, A. (20/2). Organic bee pollen: Botanical origin, nutritional value, bioactive compounds, antioxidant activity and microbiological quality. Molecules (Basel, Switzerland), 17(7), 8359-8377. https://doi.org// 0.3390/molecules I7078359

Filipiak, M., Kuszewska, K., Asselman, M., Denisow, B., Stawiarz, E., Woyciechowski, M., \& Weiner, J. (2017). Ecological stoichiometry of the honeybee: Pollen diversity and adequate species composition are needed to mitigate limitations imposed on the growth and development of bees by pollen quality. Plos One, 12(8), e0183236. https:// doi.org/I $0.1371 /$ journal.pone.0183236

Friendly, M. (20I2). HE plots for multivariate linear models. Published online: 01 Jan 2012. Journal of Computational and Graphical Statistics, I6(2), 42I-444. https://doi.org/10.1 198/ $106186007 \times 208407$

Garaffo, M. A., Vassallo-Agius, R., Nengas, Y., Lembo, E., Rando, R., Maisano, R., Dugo, G., \& Giuffrida, D. (20II) Fatty acids profile, atherogenic (IA) and thrombogenic (IT) health lipid indices, of raw roe of blue fin tuna (Thunnus thynnus L.) and their salted product "Bottarga". Food and Nutrition Sciences, 02(07), 736-743. https://doi.org//0.4236/ fns.2011.27101

Ghaeni, M., Ghahfarokhi, K. N., \& Zaheri, L. (20I3). Fatty acids profile, atherogenic (IA) and thrombogenic (IT) health lipid indices in Leiognathusbindus and Upeneussulphureus. Journal of Marine Science: Research \& Development, 3(4), 138 https://doi.org//0.4172/2155-9910.1000138

Human, H., \& Nicolson, S. W. (2006). Nutritional content of fresh, bee-collected and stored pollen of Aloe greatheadii var. davyana (Asphodelaceae). Phytochemistry, 67(14), | 486-| 492. https://doi.org//0.1016/j.phytochem.2006.05.023
Instituto Adolfo Lutz (IAL). (2008). Normas analíticas, métodos químicos e fisicos para análises de alimentos (Ist ed., p. 1020). Instituto Adolfo Lutz.

International Standards Organization - 15213 (ISO). (2003). Microbiology of food and animal feeding stuffs-Horizontal method for the enumeration of sulfite-reducing bacteria growing under anaerobic conditions. International Standards Organization.

International Standards Organization - 21527-2 (ISO). (2006). Microbiology of food and animal feeding stuffs-Horizontal method for the enumeration of yeasts and moulds - Part 2: Colony count technique in products with water activity less than or equal to 0.95 . International Standards Organization.

Jones, G. D., \& Bryant, V. M. Jr. (2004). The use of ETOH for the dilution of honey. Journal of Palynology and Aerobiology, 43, 174-182. https://doi.org//0.1080/00173/304/0019497

Kalaycioğlu, Z., Kaygusuz, H., Doker, S., Kolaylı, S., \& Erim, F. B. (2017). Characterization of Turkish honeybee pollens by principal component analysis based on their individual organic acids, sugars, minerals, and antioxidant activities. LWT, 84, 402-408. https://doi.org//0.1016/j.lwt.2017.06.003

Kaplan, M., Karaoglu, Ö., Eroglu, N., \& Silici, S. (2016). Fatty acid and proximate composition of bee bread. Food Technol. Biotechnol, 54(4), 497-504. https://doi.org/l0. | 7 | | 3/ftb.54.04.16.4635

Kjeldahl, J. (1883). New method for the determination of nitrogen. Chem. News, 48, I0I-102.

Kostić, A. Ž., Barać, M. B., Stanojević, S. P., MilojkovićOpsenica, D. M., Tešić, Ž. L., Šikoparija, B., Radišić, P., Prentović, M., \& Pešić, M. B. (20I5). Physicochemical composition and techno-functional properties of bee pollen collected in Serbia. Lwt - Food Science and Technology, 62(I), 30I-309. https://doi.org/10.1016/j.Iwt.2015.01.03।

Kroyer, G., \& Hegedus, N. (200I). Evaluation of bioactive properties of pollen extracts as functional dietary food supplement. Innovative Food Science \& Emerging Technologies, 2, |7I-174. https://doi.org/I0.10I6/SI466-8564(0I)00039-X

Lima Neto, J. S., Lopes, J. A. D., Moita Neto, J. M., Lima, S. G., Luz, C. F. P., \& Citó, A. M. G. L. (2017). Volatile compounds and palynological analysis from pollen pots of stingless bees from the mid-north region of Brazil. Brazilian Journal of Pharmaceutical Sciences, 53(2), 1-9. https://doi.org/ | 0.1590/s2 |75-979020170002/4093

Lima, D. M., Padovani, R. M., Rodriguez-Amaya, D. B., Farfán, J. A., Nonato, C. T., Lima, M. T., Salay, E., Colugnati, F. A. B., \& Galeazzi, M. A. M. (20II). Tabela brasileira de composição de alimentos/NEPA-UNICAMP (4th ed.). UNICAMP.

Louveaux, J., Maurizio, A., \& Vorwohl, G. (1978). Methods of Melissopalynology. Bee World, 59(4), 139-157. https://doi. org/I0.1080/0005772X.1978.II0977/4

Lucas, C. I. S., Andrade, W. C., Ferreira, A. F., Sodré, G. S., Carvalho, C. A. L., Costa, M. A. P. C., \& Aguiar, C. M. L. (2018). Pollen types from colonies of Melipona scutellaris Latreille, I8II (Hymenoptera: Apidae) established in a coffee plantation. Grana, 57(3), 235-2II. https://doi.org/I0. |080/00|73|34.2017.133036|

Luz, C. F. P., Fernandes-Salomão, T. M., Lage, L. G. A., Resende, H. C., Tavares, M. G., \& Campos, L. A. O. (20II). Pollen sources for Melipona capixaba Moure \& Camargo: An endangered Brazilian stingless bee. Psychem, v. Psyche: $A$ Journal of Entomology, 20II, I-7. https://doi.org//0.1I55/ $2011 / 107303$

Maia-Silva, C., Silva, C. I., Hrncir, M., Queiroz, R. T., \& Imperatriz-Fonseca, V. L. (20I2). Guia de plantas:visitadas por abelhas na Caatinga (Ist ed.). Editora Fundação Brasil Cidadão.

Marchini, L. C., Reis, V. D. A., \& Moreti, A. C. C. C. (2006). Composição físico-química de amostras de pólen coletado por abelhas Africanizadas Apis mellifera (Hymenoptera:Apidae) 
em Piracicaba, Estado de São Paulo. Ciência Rural, 36(3), 949-953. https://doi.org//0.1590/S0103-84782006000300034

Mărgăoan, R., Mărghitaş, L. A., Dezmirean, D. S., Dulf, F. V., Bunea, A., Socaci, S. A., \& Bobiş, O. (20I4). Predominant and secondary pollen botanical origins influence the carotenoid and fatty acid profile in fresh honeybee-collected pollen. Journal of Agricultural and Food Chemistry, 62(27), 6306-63/6. https://doi.org//0.1021/jf50203/8

Nascimento, A. S., Chambó, E. D., Oliveira, D. J., Andrade, B. R., Bonsucesso, J. S., \& Carvalho, C. A. L. (2018). Honey from stingless bee as indicator of contamination with metals. Sociobiology, 65(4), 727-736. https://doi.org//0.13102/ sociobiology.v65i4.3394

Nascimento, A. S., Marchini, L. C., Carvalho, C. A. L., Araújo, D. F. D., \& Silveira, T. A. (20I5). Pollen spectrum of stingless bees honey (Hymenoptera: Apidae), Paraná State, Brazil. Journal of Entomology and Zoology Studies, 3, 290-296.

Newstrom, L. E., Frankie, G. W., \& Baker, H. G. (1994). A new classification for plant phenology based on flowering pattern in low land tropical trees at La Selva, Costa Rica. Biotropica, 26(2), I4I-159. https://doi.org//0.2307/2388804

Oliveira, D. J., Carvalho, C. A. L., Sodré, G. S., Paixão, J. F., \& Alves, R. M. O. (2017). Partitioning of pollen resources by two stingless bee species in the north Bahia. Grana, 56(4), 285-289. https://doi.org// 0.1080/00173 I34.2016.1217040

Pascoal, A., Rodrigues, S., Teixeira, A., Féas, X., \& Estevinho, M. L. M. (20/4). Biological activities of commercial bee pollens: Antimicrobial, antimutagenic, antioxidant and antiinflammatory. Food and Chemical Toxicology : An International Journal Published for the British Industrial Biological Research Association, 63, 233-239. https://doi.org//0.1016/j.fct.2013. 11.010

Pita-Calvo, C., \& Vázquez, M. (2017). Differences between honeydew and blossom honeys: A review. Trends in Food Science \& Technology, 59, 79-87. https://doi.org//0.1016/j. tifs.2016.11.015

Porrini, C., Sabatini, A. G., Girotti, S., Ghini, S., Medrzycki, P., Grillenzoni, F., \& Celli, G. (2003). Honey bees and bee products as monitors of the environmental contamination. Apiacta, 38, 63-70.

Punt, W., Hoen, P. P., Blackmore, S., Nilsson, S., \& Le Thomas, A. (2007). Glossary of pollen and spore terminology. Review of Palaeobotany and Palynology, I43(I-2), I-8I. https://doi.org//0.1016/j.revpalbo.2006.06.008

$R$ Development Core Team. (2018). R: A language and environment for statistical computing. R Foundation for Statistical Computing. Retrieved Abril 20, 2018, from http://www.Rproject.org/.

Rebelo, K. S., Ferreira, A. G., \& Carvalho-Zilse, G. A. (2016). Physicochemical characteristics of pollen collected by Amazonian stingless bees. Ciência Rural, 46(5), 927-932. https://doi.org/10.1590/0103-8478cr20150999

Rodrigues, M. A. A., Keller, K. M., Keller, L. A. M., Oliveira, Á. A., Almeida, T. X., Marassi, A. C., Krueger, C. D.,
Barbosa, T. S., Lorenzon, M. C. A., \& Rosa, C. A. R. (2008). Mycological and mycotoxicological evaluation of the pollen of jataí bee (Tetragonisca angustula) from llha Grande, Angra Do Reis, RJ. Revista Brasileira de Medicina Veterinária, 30, 249-253.

Roubik, D. W., \& Moreno, J. E. P. (1991). Pollen and Spores of Barro Colorado Island. Monographs in Systematic Botany. Missouri Botanical Garden.

Santa-Bárbara, M., Machado, C. S., Sodré, G. S., Dias, L. G., Estevinho, L. M., \& Carvalho, C. A. L. (20I5) Microbiological assessment, nutritional characterization and phenolic compounds of Bee Pollen from Mellipona mandacaia Smith, 1983. Molecules (Basel, Switzerland), 20(7), |2525-I2544. https://doi.org// 0.3390/molecules2007/2525

Santos, J. F., Roppa, C., Oliveira, S. S. H., \& Valcarcel, R. (20I I). Horizontal structure and floristic composition of the shrubbyarboreal strata in forests planted to rehabilitate a degraded area of the Brazilian. Ciencia e Investigación Agraria, 38, 95-106. https://doi.org/I0.4067/S07 I8-1620201 I000100009

Silva, N. D., Junqueira, V. C. A., N. F. A, S., Taniwaki, M. H., Santos, R. F. S., \& Gomes, R. A. R. (2010). Manual de Métodos de análise microbiológica (p. 552). Ed. Varela.

Silva, F. O., Viana, B. F., \& Pigozzo, C. M. (2007). Flowering, nectar production and visiting bees of Eriope blanchetii (Lamiaceae), in sand dunes, northeastern. Iheringia. Série Zoologia, 97(I), 87-95. https://doi.org//0.1590/S007347212007000100013

Somerville, D. C. (2005). Fat Bees Skinny Bees - a manual on honey bee nutrition for beekeepers (p. 142). Rural Industries Research and Development Corporation.

Souchie, E. L., Miranda, C. C., Campello, E. F. C., Silva, E. M. R., \& Saggin-Júnior, O. J. (2005). Enrichment of regenerating forest using tree species on atlantic forest region. Floresta e Ambiente, 12, 1-6.

Souza, R. C. S., Yuyama, L. K. O., Aguiar, J. P. L., \& Oliveira, F. P. M. (2004). Nutritional value of honey and pollen of stingless bees of the Amazonian region. Acta Amazonica, 34(2), 333-336. https://doi.org//0.1590/S0044-5967200400020002 I

Viana, J. L., Francisco, A. K., Carvalho, C. A. L., \& Waldschmidt, A. M. (20I3). Genetic variability in Melipona scutellaris from Recôncavo, Bahia, Brazil. Genetics and Molecular Research: GMR, I2(3), 3444-3454. https://doi.org//0.4238/2013

Villas-Bôas, J. (2018). Aproveitamento integral dos produtos das Abelhas nativas sem ferrão (2nd ed.). ISPN.

Yang, K., Wu, D., Ye, X., Liu, D., Chen, J., \& Sun, P. (20I3). Characterization of chemical composition of Bee Pollen in China. Journal of Agricultural and Food Chemistry, 6I(3), 708-7/8. https://doi.org/10.1021/jf304056b

Zuluaga, C. M., Serrato, J. M., \& Quicazan, M. C. (20I5). Chemical, nutritional and bioactive characterization of Colombian Bee-Bread. Chemical Engineering Transactions, 43, 175-180. https://doi.org//0.3303/CETI543030 